\title{
Primary central nervous system Hodgkin Lymphoma: A case discussion and a hypothesis on the etiology
}

\author{
Ahmad Alfaseh, Mhd Nabeel Rajeh'1, Ghiath Hamed²
}

Department of Urology, Kidney Surgical Hospital, Damascus, Syria, ${ }^{1}$ Division of Hematology and Medical Oncology, Saint Louis University School of Medicine, St. Louis, MO, ${ }^{2}$ Hematogenix Laboratory Services, Tinley Park, IL, USA

\begin{tabular}{|c|}
\hline Access this article online \\
\hline Website: www.avicennajmed.com \\
\hline DOI: 10.4103/ajm.AJM_104_18 \\
\hline Quick Response Code: \\
\hline
\end{tabular}

ABSTRACT
Hodgkin Lymphoma (HL) is a systemic disease with involvement of the cervical, supraclavicular,
and mediastinal lymph nodes. It is commonly diagnosed in patients within the second and
third decades of their lives. Diagnosis is usually made based on the distinct morphological
and immunohistochemical characteristics, with the tissue biopsy being the cornerstone of
workup. Extranodal presentation of $\mathrm{HL}$ is unusual and seldom encountered. Primary HL of the
central nervous system (CNS) is exceedingly rare. We herein report a case of a 38-year-old
male patient who was diagnosed with primary CNS-HL. The patient was treated with complete
surgical resection followed by radiotherapy and chemotherapy. The patient was disease-free for
7 years postoperatively without any clinical evidence of relapse. We also discussed a possible
role of CNS regulatory T-cells (Tregs) in developmental primary CNS-HL.
Key words: Central nervous system lymphoma, cerebellar tumor, Hodgkin Lymphoma,
T-regulatory cells

\section{INTRODUCTION}

Hodgkin Lymphoma (HL) is a unique hematopoietic neoplasm characterized by the presence of neoplastic Reed-Sternberg (RS) cells in an inflammatory background. Central nervous system (CNS) involvement in systemic HL is fairly uncommon, with incidence as low as $0.02 \%$ of all HL cases, most of which are due to relapsing systemic involvement or progressive disease. ${ }^{[1]}$ Primary CNS-HL is even more rare with only 22 cases have been reported in the literature and summarized by Cecyn et al. ${ }^{[2]}$ In this report, we present a primary CNS-HL in an immunocompetent patient without evidence of disease recurrence after 7 years of follow-up.

\section{CASE REPORT}

A 38-year-old Caucasian gentleman with no prior significant history presented with headache, vomiting, and frequent falls over the course of 2 months. Physical examination revealed normal vital signs. Neurological assessment showed left-sided ataxia, dysmetria, muscular hypotonia,

Address for correspondence: Dr. Ahmad Alfaseh,

Department of Urology, Kidney Surgical Hospital, Damascus, Syria.

E-mail: dr.ahmadalfaseh@gmail.com and difficult in coordination movements. No evidence of peripheral lymphadenopathy or hepatosplenomegaly was noted on physical examination.

Routine laboratory analysis showed no abnormal findings. Magnetic resonance imaging (MRI) with intravenous contrast revealed an intra-axial lesion $(13.7 \mathrm{~mm} \times 23.5 \mathrm{~mm} \times 21.1 \mathrm{~mm})$ compressing the fourth ventricular and leading to surrounding vasogenic edema and mass effect in the left cerebellar hemisphere. A homogenous hyperintensity was also seen postcontrast without central necrosis [Figure 1]. Differential diagnosis included a primary brain tumor, metastasis from other sites, and lymphoma. Computed tomography (CT) scan of the neck, chest, abdomen, and pelvis did not reveal any significant abnormalities.

This is an open access journal, and articles are distributed under the terms of the Creative Commons Attribution-NonCommercial-ShareAlike 4.0 License, which allows others to remix, tweak, and build upon the work non-commercially, as long as appropriate credit is given and the new creations are licensed under the identical terms.

For reprints contact: reprints@ medknow.com

Cite this article as: Alfaseh A, Rajeh MN, Hamed G. Primary central nervous system Hodgkin Lymphoma: A case discussion and a hypothesis on the etiology. Avicenna J Med 2019;9:28-31 
In light of previous findings, the patient was deemed a candidate for surgical resection of tumor and underwent complete tumor resection.

Histopathological examination of the mass using hematoxylin and eosin-stained sections showed mixed cell background with multiple scattered RS cells. The RS cells were immunoreactive for CD30 marker and were negative for CD20 and ALK-1 markers. CD3 and CD5 markers highlight the small background lymphocytes [Figure 2]. The overall findings were most compatible with classical HL, mixed cellularity type.

Bone marrow biopsy was also obtained for staging and was found to be normal. Lumbar puncture revealed clear cerebrospinal fluid (CSF) with normal protein, glucose, and white blood cell count. No malignant cells were seen on microscopic examination of centrifuged CSF sample. HIV test was negative.

Postoperative course was uneventful. The patient received whole-brain radiation with a dose of 4000 centigray (cGy) followed by a boost of $1000 \mathrm{cGy}$, which was targeted toward the resection site. Chemotherapy containing two courses of adriamycin, bleomycin, vinblastine, and dacarbazine was given to prevent tumor recurrence and metastasis.

The patient was followed regularly with routine physical and neurological examination, laboratory tests (complete blood count, erythrocyte sedimentation rate, and lactate dehydrogenase), MRI of the brain, and low-dose CT scan of the neck, chest, abdomen, and pelvis at 3, 6, 12, 24, and 36 months postoperatively [Figure 3]. No evidence of recurrence was noted over the course of 7 years.

\section{DISCUSSION}

CNS has been commonly thought of as an immune-isolated organ without any connection with the peripheral immune system. However, recent discoveries showed that the brain has an active lymphatic network presents in the meninges, with heavy immunocytes (lymphocytes, monocytes, macrophages, and dendritic cells) shift from these lymph vessels to the CNS tissue through the blood-brain barrier. This movement helps the peripheral immune system perform active surveillance inside the CNS, even in normal physiologic conditions. ${ }^{[3]}$

While the CNS is rich in lymphocytes, the incidence of $\mathrm{HL}$ in CNS remains exceedingly rare with only 22 cases reported in the literature and summarized by Cecyn et al. ${ }^{[2]}$ which raises the questions about the role of predisposing in the
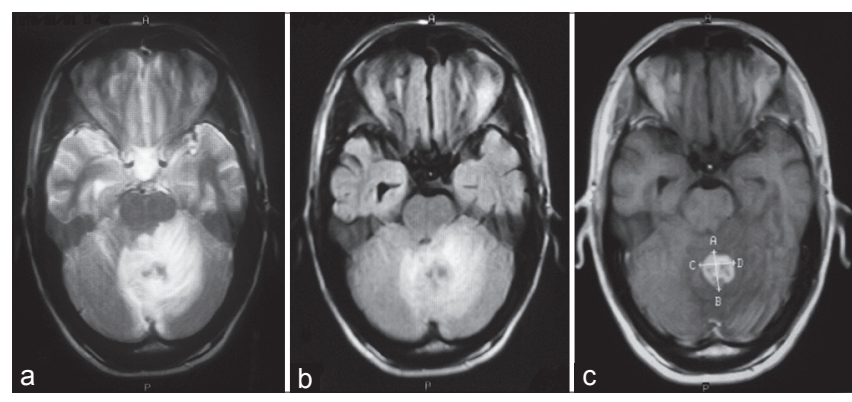

Figure 1: Preoperative magnetic resonance imaging images. (a) T2-weighted axial, (b) T1-weighted axial, (c) Gd-enhanced T1-weighted axial. Images show contrast-enhancing lesion surrounded by a vasogenic edema with a little compress to fourth ventricular

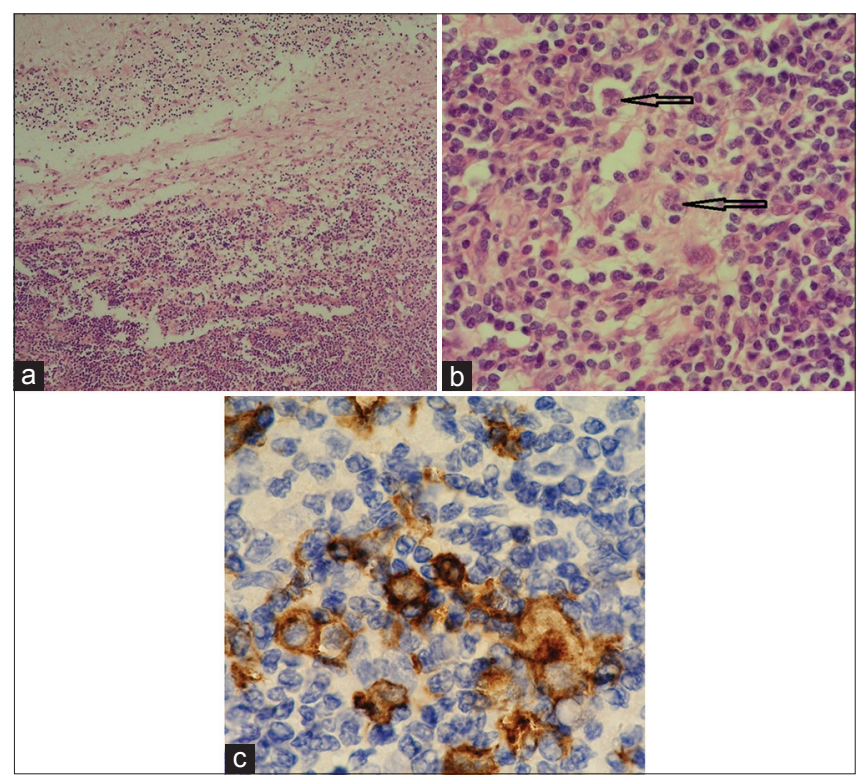

Figure 2: Histopathologic findings. (a) A hematoxylin and eosin section shows a tumor tissue within cerebellar tissue, (b) a hematoxylin and eosin section shows Reed-Sternberg cells (arrows), eosinophils, plasma cells, histiocytes, and small lymphocytes, (c) immunostain shows immunoreactivity for CD30 marker
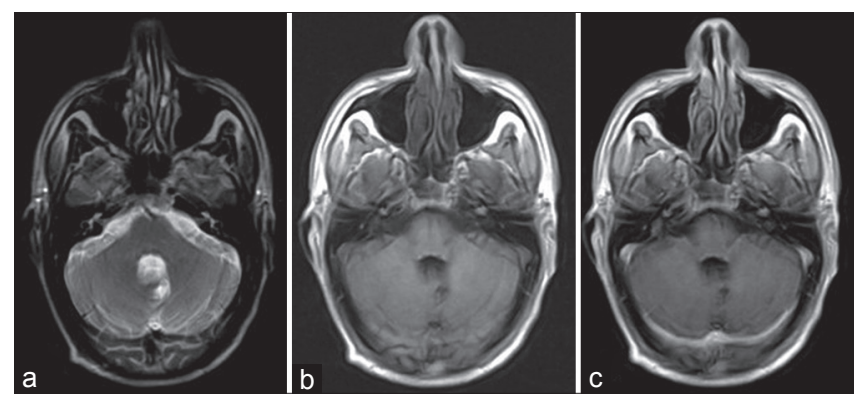

Figure 3: Follow-up magnetic resonance imaging 36 months after operation (a) T2-weighted axial, (b) T1-weighted axial, (c) Gd-enhanced T1-weighted axial. Images show preoperative-enhanced mass is removed totally. There is no evidence of local recurrence, edema, or abnormal enhancement

development of primary CNS-HL. In the CNS, immunocytes reside under a rigid control from CNS Tregs, specifically CD25+ T-cells which are capable of restricting proliferation and cytokines production from these immunocytes. Impaired function of the CNS Tregs leads to negative consequences. 
For example, if this function is decreased, this may activate autoreactive T- and B-lymphocytes in the CNS leading to an autoimmune reaction similar to that in multiple sclerosis, or increased neurodegeneration due to excessive immune response similar to what's observed in Parkinson's disease. ${ }^{[3,4]}$ Other studies suggested that overactivation of Tregs or increase their numbers may weaken the immune response and antitumor immunity and might favor tumor development or growth. ${ }^{[3,5]}$ In addition, recent studies showed that the number and activity of Tregs (especially $\mathrm{CD} 4+\mathrm{CD} 25+$ ) in the HL tissues are very high, and it would be expected to exert profound local immunosuppressive effects by inhibiting both interleukin-2 (IL-2) production and the upregulation of IL-2 Ra-chain (CD25) expression, thus delaying or blocking the activation of CD8+ and natural killer cells to tumor antigens, which leads to protection of RS cells from immunological surveillance and clearance. ${ }^{[-9]}$ The presence of Tregs in the microenvironment around RS may have a prognostic value. Furthermore, some reports suggested that the increased numbers of Tregs might have a positive prognostic value in $\mathrm{CHL},{ }^{[10]}$ while others found the prognostic value to be dependent on the ratio of Tregs to other background infiltrating cells rather than its absolute numbers. ${ }^{[1]}$ The high ratio of Tregs in the microenvironment around RS cells over T-helper 2 is associated with significantly shortened disease-free survival, while a low ratio improved disease-free survival, which supports the suggestion of the inhibitory effects on antitumor immune response mediated by Tregs. ${ }^{[12]}$ The paucity of primary CNS-HL cases makes it more difficult to identify the risk factors associated with the development of this malignancy. Family history of HL and male gender have been considered risk factors. ${ }^{[13]}$ The role of immunosuppression and Epstein-Barr virus infection in systemic and primary CNS-HL have also been suggested. ${ }^{[14]}$ The presence of EBV in RS cells correlates with increased Tregs (CD4+CD25+FOXP3+) quantification in the tumor microenvironment and decreases the antitumor immunity toward RS. Fortunately, the presence of EBV with HL does not impact the survival in these patients. ${ }^{[15]}$ Better understanding of the role of Tregs in HL pathogenesis will help in development of more efficient therapies with less toxicity than the traditional radiation and chemotherapy, especially in cases of CNS-HL. For example, adoptive transfer of cytotoxic lymphocytes may be more effective if combined with measures to overcome this regulatory cell activity. ${ }^{[8]}$

\section{CONCLUSION}

We report an exceedingly rare case of primary CNS-HL in an immunocompetent patient, with no evidence of systemic involvement found after the staging process. In this report, we also discuss the role of Tregs in the CNS immune system.
While little is known about etiology, optimal management, and the prognosis of such case, we discuss the role of impaired function of Tregs in the CNS in the development and progression primary CNS HLs. Further insight into the pathophysiology will help establish unique and less toxic treatment for such cases compared to traditional therapy used in HLs.

\section{Declaration of patient consent}

The authors certify that they have obtained all appropriate patient consent forms. In the form the patients has/have given their consent for their images and other clinical information to be reported in the journal. The patients understand that their names and initials will not be published and due efforts will be made to conceal their identity, but anonymity cannot be guaranteed.

\section{Financial support and sponsorship}

Nil.

\section{Conflicts of interest}

There are no conflicts of interest.

\section{REFERENCES}

1. Re D, Fuchs M, Schober T, Engert A, Diehl V. CNS involvement in Hodgkin's lymphoma. J Clin Oncol 2007;25:3182.

2. Cecyn KZ, Chaves EM, Oliveira JS. Primary central nervous system involvement in classical Hodgkin's lymphoma: Case report and review of the literature. J Blood Lymph 2017;8:196. doi:10.4172/21657831.1000196.

3. Negi N, Das BK. CNS: Not an immunoprivilaged site anymore but a virtual secondary lymphoid organ. Int Rev Immunol 2018;37:57-68.

4. Reynolds AD, Banerjee R, Liu J, Gendelman HE, Mosley RL. Neuroprotective activities of CD4+CD25+ regulatory $T$ cells in an animal model of Parkinson's disease. J Leukoc Biol 2007;82:1083-94.

5. Ha TY. The role of regulatory $T$ cells in cancer. Immune Netw 2009;9:209-35.

6. Piccirillo CA, Shevach EM. Cutting edge: Control of CD8 + T cell activation by CD4+CD25+ immunoregulatory cells. J Immunol 2001;167:1137-40.

7. Wolf AM, WolfD, Steurer M, Gastl G, Gunsilius E, Grubeck-Loebenstein B, et al. Increase of regulatory $\mathrm{T}$ cells in the peripheral blood of cancer patients. Clin Cancer Res 2003;9:606-12.

8. Marshall NA, Christie LE, Munro LR, Culligan DJ, Johnston PW, Barker $\mathrm{RN}$, et al. Immunosuppressive regulatory $\mathrm{T}$ cells are abundant in the reactive lymphocytes of Hodgkin lymphoma. Blood 2004;103:1755-62.

9. Alvaro T, Lejeune M, Salvadó MT, Bosch R, García JF, Jaén J, et al. Outcome in Hodgkin's lymphoma can be predicted from the presence of accompanying cytotoxic and regulatory $\mathrm{T}$ cells. Clin Cancer Res 2005; 11:1467-73.

10. Tzankov A, Meier C, Hirschmann P, Went P, Pileri SA, Dirnhofer S, et al. Correlation of high numbers of intratumoral FOXP3 + regulatory T cells with improved survival in germinal center-like diffuse large B-cell lymphoma, follicular lymphoma and classical Hodgkin's lymphoma. Haematologica 2008;93:193-200.

11. Badoual C, Hans S, Fridman WH, Brasnu D, Erdman S, Tartour E, et al. Revisiting the prognostic value of regulatory $T$ cells in patients with 
cancer. J Clin Oncol 2009;27:e5-6.

12. Schreck S, Friebel D, Buettner M, Distel L, Grabenbauer G, Young LS, et al. Prognostic impact of tumour-infiltrating th 2 and regulatory $\mathrm{T}$ cells in classical Hodgkin lymphoma. Hematol Oncol 2009;27:31-9.

13. van Blydenstein SA, Patel M, Philip V, Lakha A, Pather S, Westgarth-Taylor T, et al. Classical Hodgkin lymphoma involving the central nervous system (brain) - an unusual presentation. Clin Case Rep 2014;2:88-92.
14. Gerstner ER, Abrey LE, Schiff D, Ferreri AJ, Lister A, Montoto S, et al. CNS Hodgkin lymphoma. Blood 2008;112:1658-61.

15. Assis MC, Campos AH, Oliveira JS, Soares FA, Silva JM, Silva PB, et al. Increased expression of CD4+CD25+FOXP3+ regulatory $T$ cells correlates with Epstein-Barr virus and has no impact on survival in patients with classical Hodgkin lymphoma in Brazil. Med Oncol 2012;29:3614-9. 